\title{
Optical design of two-reflector systems, the Monge-Kantorovich mass transfer problem and Fermat's principle
}

\author{
Tilmann Glimm \& Vladimir Oliker \\ Department of Mathematics and Computer Science, \\ Emory University, Atlanta, Georgia 30322
}

May 16, 2002

\begin{abstract}
It is shown that the problem of designing a two-reflector system transforming a plane wave front with given intensity into an output plane front with prescribed output intensity can be formulated and solved as the Monge-Kantorovich mass transfer problem ${ }^{1}$.
\end{abstract}

\section{Introduction}

Consider a two-reflector system of configuration shown schematically on Fig. 1. Let $\left(x=\left(x_{1}, x_{2}, \ldots, x_{n}\right), z\right)$ be the Cartesian coordinates in $\mathbb{R}^{n+1}, n \geq$ 2 , with $z$ being the horizontal axis and $x_{1}, x_{2}, \ldots, x_{n}$ the coordinates in the hyperplane $\alpha: z=0$. Let $B_{1}$ denote a beam of parallel light rays propagating in the positive $z$-direction and let $\bar{\Omega}$ denote the wavefront which is the cross section of $B_{1}$ by hyperplane $\alpha$. Assume that $\Omega$ is a bounded domain on $\alpha$. An individual ray of the front is labeled by a point $x \in \bar{\Omega}$. The light intensity of the beam $B_{1}$ is denoted by $I(x), x \in \Omega$, where $I$ is a non-negative integrable function.

${ }^{1} 2000$ Mathematics Subject Classification: 35J65, 78A05, 49K20 


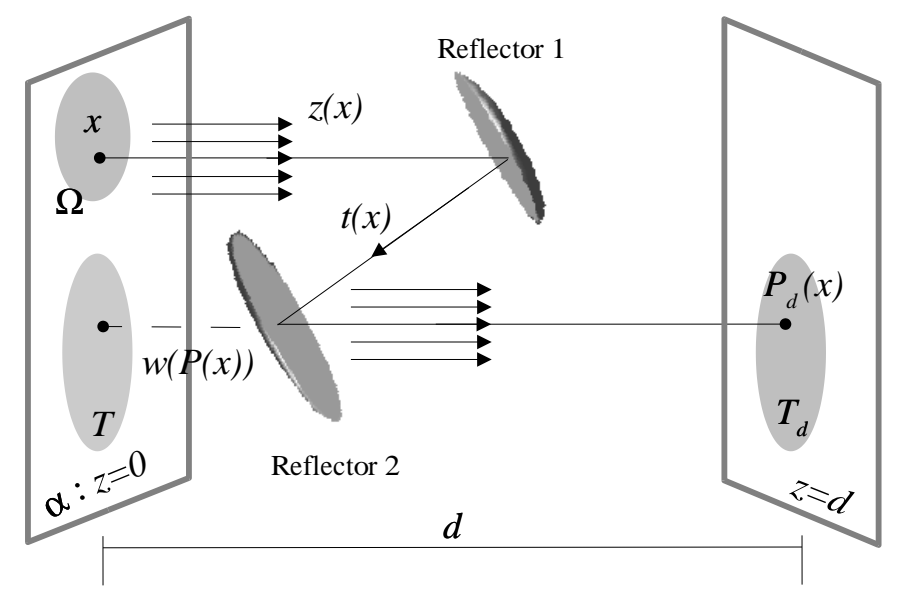

Figure 1: Sketch for Problem I

The incoming beam $B_{1}$ is intercepted by the first reflector $R_{1}$, defined as a graph of a function $z(x), x \in \bar{\Omega}$. The rays in $B_{1}$ are reflected off $R_{1}$ forming a beam of rays $B_{2}$. The beam $B_{2}$ is intercepted by reflector $R_{2}$ which transforms it into the output beam $B_{3}$. The beam $B_{3}$ also consists of parallel light rays propagating in the same direction as $B_{1}$. The output wavefront at a distance $d>0$ from the hyperplane $\alpha$ is denoted by $\bar{T}_{d}$; the projection of $\bar{T}_{d}$ on the hyperplane $\alpha$ we denote by $\bar{T}$. The second reflector $R_{2}$ is also assumed to be a graph of a function $w(p), p \in \bar{T}$. The quantity $\frac{1}{\mid J\left(P_{d}(x) \mid\right.}$, where $P_{d}$ is the map of $\bar{\Omega}$ on $\bar{T}_{d}$ and $J$ is the Jacobian, is the expansion ratio and it measures the expansion of a tube of rays due to the two reflections [5]. It is assumed that both $R_{1}$ and $R_{2}$ are perfect reflectors and no energy is lost in the transformation process. Consequently, the corresponding relation between the input intensity $I$ on $\Omega$ and output intensity $L$ on $T_{d}$ is given by

$$
L\left(P_{d}(x)\right) \mid J\left(P_{d}(x) \mid=I(x) .\right.
$$

The "two-reflector" problem that needs to be solved by designers of optical systems consists in determining the reflectors $R_{1}$ and $R_{2}$ so that all of the properties of the two-reflector system above hold for prescribed in advance domains $\Omega, T$ and positive integrable functions $I(x), x \in \Omega$, and $L(p), p \in T$; 
see Malyak [6] and other references there. It is usually assumed in applications that $\Omega$ and $T_{d}$ are bounded and convex.

Two fundamental principles of geometrical optics are used to describe the transformation of the beam $B_{1}$ into beam $B_{3}$ : the classical reflection law leading to the ray tracing equations defining the map $P_{d}$ and the energy conservation law for the energy flux along infinitesimally small tubes of rays; see [6] where the problem is formulated for rotationally symmetric data and a class of rotationally symmetric solutions is found.

The problem of recovering reflectors $R_{1}$ and $R_{2}$ without assuming rotational symmetry was formulated rigorously by Oliker and Prussner in [7], and it was shown that it can be considered as a problem of determining a special map of $\bar{\Omega} \rightarrow \bar{T}$ with a potential satisfying an equation of Monge-Ampère type relating the input and output intensities. Existence and uniqueness of weak solutions were established by Oliker at that time but only the numerical results implementing a constructive scheme for proving existence were presented in [7] for several test cases. Detailed proofs were given in [8].

In this paper we show that this problem can also be studied in the framework of the Monge-Kantorovich mass transfer problem studied by Brenier [2], Caffarelli [3], Gangbo and McCann [4], and other authors. In our notation, the Monge-Kantorovich mass transfer problem is to transfer the intensity $I$ on $\Omega$ into the intensity $L$ on $T$ via a map $P: \Omega \rightarrow T$ for which the total transportation cost $\int_{\Omega} C(x, P(x)) I d x$ is minimized. Here $C(x, p)$ is a given strictly convex cost function.

The proof of existence and uniqueness of solutions to the MongeKantorovich problem is obtained by solving a minimization problem for the functional

$$
(\zeta, \omega) \mapsto \int_{\Omega} \zeta I d x-\int_{T} \omega L d p
$$

considered on pairs of continuous functions $\zeta$ on $\bar{\Omega}$ and $\omega$ on $\bar{T}$ that satisfy

$$
\zeta(x)-\omega(p) \geq-C(x, p), \quad x \in \bar{\Omega}, p \in \bar{T} .
$$

Under various conditions it is shown in [2], [3], [4] that this functional is minimized by some pair $\left(\zeta_{0}, \omega_{0}\right)$ (referred to as Kantorovich potentials), and that $P(x)=x+\nabla \zeta_{0}$ solves the Monge-Kantorovich problem.

Applying these ideas, we show that the geometric optics problem at hand can be formulated as a Monge-Kantorovich mass transfer problem with a 
quadratic cost function; see section 6. The Kantorovich potentials correspond to the pair of reflectors that solve the problem. The condition (3) has a geometric meaning; namely, it filters out reflectors that allow only optical paths longer than a certain prescribed one. The functional (2) to be minimized is the mean horizontal distance between points of the two reflectors, with the average weighted by the two intensities.

We prove that there are always two different reflectors system satisfying the stated requirements. The corresponding ways in which one intensity is transfered into the other one are exactly the most and the least energy efficient in the sense of the Monge-Kantorovich cost. This result is thus ultimately a variant of Fermat's principle.

The fact that the solution to the above geometrical optics problem can be derived from a variational principle gives rise to a numerical treatment of the problem different from the one suggested in [8]. In particular, when the problem of minimizing (2) under constraints (3) is discretized we have the linear programming problem with quadratic constraints. We ran some numerical experiments with this approach and intend to return to this point in a separate publication.

This paper is organized as follows. In section 2, we recall some results from [8] concerning the ray tracing map, assuming smoothness of the reflectors, and formulate the main "two-reflector" problem. In section 3, we give a geometric characterization of reflectors as envelopes of certain families of paraboloids. Such characterization is of independent interest. In section 4 we use this geometric characterization to define weak solutions of type A and type B of the two-reflector problem. To prove existence and uniqueness of solutions for each type we utilize the ideas of the Monge-Kantorovich theory and introduce the functional (2) on a certain class of "quasi-reflector" systems. This is done in section 5. In the same section it is shown that the problem of finding weak solutions of type $\mathrm{A}$ is equivalent to finding minimizers of (2). Weak solutions of type B correspond to maximizers of (2). On the other hand, existence of minimizers (maximizers) to this functional is not difficult and has been established before in [2], [3], [4]. This implies existence of solutions. The uniqueness in respective class is established in section 6 by proving that the ray tracing map $\tilde{P}$ associated with a weak solution minimizes or maximizes the quadratic Monge-Kantorovich cost for which the functional (2) is the dual. The main theorem on existence and uniqueness of weak solutions to the two-reflector problem is stated and proved in section 7. 
Finally, we note that same methods lead to variational formulations and solutions of other geometrical optics problems involving systems with single and multiple reflectors.

\section{Statement of the problem}

We begin by reviewing briefly the analytic formulation of the problem for smooth reflectors; see [8] for more details.

Let $R_{1}$ be given by the position vector $r_{1}(x)=(x, z(x)), x \in \bar{\Omega}$, with $z \in C^{2}(\bar{\Omega})$. The unit normal $u$ on $R_{1}$ is given by

$$
u=\frac{(-\nabla z, 1)}{\sqrt{1+|\nabla z|^{2}}} .
$$

Consider a ray labeled by $x \in \bar{\Omega}$ and propagating in the positive direction $k$ of the $z$ - axis. According to the reflection law the direction of the ray $y(x)$ reflected off $R_{1}$ is given by

$$
y=k-2\langle k, u\rangle u=k-2 \frac{(-\nabla z, 1)}{1+|\nabla z|^{2}},
$$

where $\langle$,$\rangle is the inner product in R^{n+1}$. Denote by $t(x)$ the distance from reflector $R_{1}$ to reflector $R_{2}$ along the ray reflected in the direction $y(x)$ and let $s(x)$ be the distance from $R_{2}$ to the wavefront $\bar{T}_{d}$ along the corresponding ray reflected off $R_{2}$. Assume for now that $t \in C^{1}(\bar{\Omega})$ and $R_{2}$ is a $C^{1}$ hypersurface. The total optical path length (OPL) corresponding to the ray associated with the point $x \in \bar{\Omega}$ is $l(x)=z(x)+t(x)+s(x)$. A calculation shows that $l(x)=$ const $\equiv l$ on $\bar{\Omega}$. Since

$$
R_{2}: \quad r_{2}(x)=r_{1}(x)+t(x) y(x), \quad x \in \bar{\Omega},
$$

the image of $x$ on the reflected wavefront $\bar{T}_{d}$ is given by

$$
P_{d}(x)=r_{1}(x)+t(x) y(x)+s(x) k, \quad x \in \bar{\Omega} .
$$

The equation (5) is the ray tracing equation for this two-reflector system.

Introduce the map $P(x)=P_{d}(x)-d k: \bar{\Omega} \rightarrow \bar{T}$. A calculation [8] shows that

$$
p=P(x)=x+\beta \nabla z(x), \quad x \in \bar{\Omega},
$$


where $\beta=l-d$ is the "reduced" optical path length.

To simplify the notation we will write $L(P(x))$ instead of $L\left(P_{d}(x)\right)$ (三 $L(P(x)+d k))$. For the input intensity $I(x), x \in \Omega$, and the output intensity $L(P(x))$ on $T_{d}$ we have in accordance with the differential form of the energy conservation law (1),

$$
L(P(x))|J(P(x))|=I(x), \quad x \in \Omega,
$$

where we also take into account that $J\left(P_{d}\right)=J(P)$. It follows from (7) that $\Omega, T, I$ and $L$ must satisfy the necessary condition

$$
\int_{T} L(p) d p=\int_{\Omega} I(x) d x
$$

It follows from (6) that $J(P)=\operatorname{det}[\operatorname{Id}+\beta \operatorname{Hess}(z)]$, where $I d$ is the identity matrix and Hess is the Hessian. Hence, by (7),

$$
L(x+\beta \nabla z)|\operatorname{det}[I d+\beta H \operatorname{ess}(z)]|=I \text { in } \Omega .
$$

Thus, the problem of determining the reflectors $R_{1}$ and $R_{2}$ with properties described in the introduction requires solving the following

Problem I. Given bounded domains $\Omega$ and $T$ on the hyperplane $\alpha$ and two nonnegative, integrable functions $I$ on $\Omega$ and $L$ on $T$ satisfying (8), it is required to find a function $z \in C^{2}(\bar{\Omega})$ such that the map

$$
P_{\alpha}=x+\beta \nabla z: \bar{\Omega} \rightarrow \bar{T}
$$

is a diffeomorphism satisfying equation (9).

It is shown in [7] that once such a function $z$ is found, the function $w$ describing the second reflector is determined by $z$ and $\beta$ as

$$
w(P(x))=d-s(x)=z(x)+\frac{\beta}{2}\left(|\nabla z|^{2}-1\right) .
$$

Following [8] we introduce the function

$$
V(x)=\frac{x^{2}}{2}+\beta z(x)-\frac{\beta^{2}}{2} .
$$


Then by (6) and (11)

$$
\begin{array}{r}
P=\nabla V, \\
w=\frac{1}{\beta}\left[V-\langle x, \nabla V\rangle+\frac{1}{2}|\nabla V|^{2}\right],
\end{array}
$$

where $V-\langle x, \nabla V\rangle$ is the negative of the usual Legendre transform of $V$. Thus, $V$ is a potential for the map $P: \bar{\Omega} \rightarrow \bar{T}$. If $P$ is a diffeomorphism then the inverse of the transformation $(x, z(x)) \rightarrow(p, w(p))$, where $p=P(x)$, is given by

$$
\begin{array}{r}
x(p)=P^{-1}(p)=p-\beta \nabla_{p} w(p), \\
\left.z(p)=w(p)-\frac{\beta}{2}\left|\nabla_{p} w(p)\right|^{2}+\frac{\beta}{2}\right), \quad p \in \bar{T} .
\end{array}
$$

In terms of the potential $V$ the equation (9) becomes

$$
L(\nabla V)|\operatorname{det} H \operatorname{ess}(V)|=I \text { in } \Omega,
$$

which is an equation of Monge-Ampère type.

In order to clarify the relations between the parameters $l, d$ and $\beta$, note first that it is the reduced optical path length $\beta$ that is intrinsic to the problem. The choice of cross sections of the fronts (that is, the selection of a particular value for $d)$ is extraneous. In fact, it is easy to see that if $(z, w)$ are two reflectors as above and we change $d$ to $d^{\prime}$ then $l^{\prime}-d^{\prime}=l-d=\beta$ and $(z, w)$ are not affected by such change.

Finally, we note that the two-reflector system described above has the following two symmetries. For $\lambda \in \mathbb{R}^{+}$put $z^{\prime}(x)=\lambda z(x)$ and $\beta^{\prime}=\frac{1}{\lambda} \beta$. Then

$$
\begin{array}{r}
P^{\prime}(x)=P(x), \\
w^{\prime}(p)=\lambda w(p)+\frac{\beta}{2}\left(\lambda-\frac{1}{\lambda}\right),
\end{array}
$$

where $P^{\prime}(x)=x+\beta^{\prime} \nabla z^{\prime}(x)$. In other words, the system is invariant under some combination of flattening (stretching) the first and translating and flattening (stretching) the second reflector.

Note also that a horizontal translation of both reflectors, that is, adding the same constant to $z$ and $w$, does not change $\beta$ and the map $P$. 


\section{Geometric characterization of reflectors}

We examine first more closely the relationship between the functions $z, V$ and $w$ for smooth reflectors. For the rest of the paper we assume that $\Omega$ and $T$ are bounded domains on the hyperplane $\alpha$. We continue to assume that the map $P: \bar{\Omega} \rightarrow \bar{T}$ is a diffeomorphism. Let $x \in \bar{\Omega}, p \in \bar{T}$ and

$$
Q(x, p)=\langle x, p\rangle+\beta w(p)-\frac{p^{2}}{2} .
$$

If $p=P(x)$ then by (12) - (14) we have

$$
V(x)=Q(x, P(x)) .
$$

Denote by $S_{V}$ the graph of $V$ over $\bar{\Omega}$. Let $x_{0} \in \bar{\Omega}$ and $p_{0}=P\left(x_{0}\right)$. The tangent hyperplane to $S_{V}$ at $\left(x_{0}, V\left(x_{0}\right)\right)$ is given by the equation

$$
Z=\left\langle x, p_{0}\right\rangle-\left\langle x_{0}, p_{0}\right\rangle+V\left(x_{0}\right),
$$

where $(x, Z)$ denotes an arbitrary point on that hyperplane. Taking into account (14), we obtain

$$
\left\langle x, p_{0}\right\rangle-\left\langle x_{0}, p_{0}\right\rangle+V\left(x_{0}\right)=\left\langle x, p_{0}\right\rangle+\beta w\left(p_{0}\right)-\frac{p_{0}^{2}}{2}=Q\left(x, p_{0}\right) .
$$

Since $V\left(x_{0}\right)=Q\left(x_{0}, p_{0}\right)$, we conclude that $Z=Q\left(x, p_{0}\right)$ is the tangent hyperplane to $S_{V}$ at $\left(x_{0}, V\left(x_{0}\right)\right)$. Consequently, if $V$ is convex then $Z=$ $Q\left(x, p_{0}\right)$ is a supporting hyperplane to $S_{V}$ from below and if $V$ is concave then $Z=Q\left(x, p_{0}\right)$ is supporting to $S_{V}$ from above (relative to positive direction of the $z$-axis). Because $\bar{T}$ is bounded there are no vertical tangent hyperplanes to the graph of $V$ and we have

$$
\begin{gathered}
V(x) \geq Q\left(x, p_{0}\right) \text { for all } x \in \bar{\Omega} \text { if } V \text { is convex, } \\
V(x) \leq Q\left(x, p_{0}\right) \text { for all } x \in \bar{\Omega} \text { if } V \text { is concave. }
\end{gathered}
$$

Since for every $p \in \bar{T}$ the hyperplane $Q\left(x^{\prime}, p\right)$ is supporting to $S_{V}$ at some $\left(x^{\prime}, V\left(x^{\prime}\right)\right)$, we get

$$
\begin{aligned}
& V(x) \geq Q(x, p) \text { for all } x \in \bar{\Omega}, p \in \bar{T} \text { if } V \text { is convex, } \\
& V(x) \leq Q(x, p) \text { for all } x \in \bar{\Omega}, p \in \bar{T} \text { if } V \text { is concave }
\end{aligned}
$$


and in both cases we have equalities if $p=P(x)$.

Let

$$
U(p)=\frac{p^{2}}{2}-\beta w(p)-\frac{\beta^{2}}{2}, R(x, p)=\langle x, p\rangle-\beta z(x)-\frac{x^{2}}{2} .
$$

It follows from (19) and (20) that

$$
\begin{aligned}
& U(p) \geq R(x, p) \text { for all } x \in \bar{\Omega}, p \in \bar{T} \text { if } V \text { is convex, } \\
& U(p) \leq R(x, p) \text { for all } x \in \bar{\Omega}, p \in \bar{T} \text { if } V \text { is concave }
\end{aligned}
$$

and in both cases equalities are achieved if $p=P(x)$. Also, for any fixed $x_{0} \in \bar{\Omega}$ the hyperplane $R\left(x_{0}, p\right)$ is supporting to the graph $S_{U}$ of $U$ at $\left(p_{0}=\right.$ $\left.P\left(x_{0}\right), U\left(p_{0}\right)\right)$.

Using the usual characterization of convex functions [10] we obtain from (19), (21) and (20), (22)

$$
\begin{aligned}
& V(x)=\sup _{p \in \bar{T}} Q(x, p), \quad U(p)=\sup _{x \in \bar{\Omega}} R(x, p) \text { when } V \text { is convex, } \\
& V(x)=\inf _{p \in \bar{T}} Q(x, p), \quad U(p)=\inf _{x \in \bar{\Omega}} R(x, p) \text { when } V \text { is concave. }
\end{aligned}
$$

For convex $V$ this implies

$$
\begin{aligned}
& z(x)=\sup _{p \in \bar{T}}\left[\frac{\beta^{2}-|x-p|^{2}}{2 \beta}+w(p)\right], \quad x \in \bar{\Omega} . \\
& w(p)=\inf _{x \in \bar{\Omega}}\left[\frac{|x-p|^{2}-\beta^{2}}{2 \beta}+z(x)\right], \quad p \in \bar{T} .
\end{aligned}
$$

Similarly, when $V$ is concave we have

$$
\begin{aligned}
& z(x)=\inf _{p \in \bar{T}}\left[\frac{\beta^{2}-|x-p|^{2}}{2 \beta}+w(p)\right], \quad x \in \bar{\Omega} . \\
& w(p)=\sup _{x \in \bar{\Omega}}\left[\frac{|x-p|^{2}-\beta^{2}}{2 \beta}+z(x)\right], \quad p \in \bar{T} .
\end{aligned}
$$

The characterizations (23), (24) and (25), (26) have a simple geometric meaning. To describe it, consider first the case when $V$ is convex. Recall that the total optical path length $l=z(x)+t(x)+d-w(P(x))=$ const (see 
Fig. 1). Also, $t^{2}(x)=|x-P(x)|^{2}+\mid z(x)-w\left(\left.P(x)\right|^{2}\right.$ and $\beta=l-d$. It follows from $(23)$ that $(x, z(x))$ is a point on the graph of the paraboloid

$$
k_{p, w}(x)=\frac{\beta^{2}-|x-p|^{2}}{2 \beta}+w, \quad x \in \alpha,
$$

with the focus at $(p=P(x), w(P(x)))$ and focal parameter $\beta$.

Similarly, it follows from (24) that a point $(p=P(x), w(P(x)))$ on the second reflector lies on a paraboloid

$$
h_{x, z}(p)=\frac{|x-p|^{2}-\beta^{2}}{2 \beta}+z, \quad p \in \alpha,
$$

with the focus at $(x, z(x))$ and focal parameter $\beta$.

Let $K_{p, w(p)}$ be the convex body bounded by the graph of paraboloid $k_{p, w}(x)$ and $H_{x, z(x)}$ the convex body bounded by the graph of paraboloid $h_{x, z}(p)$. Then (23) and (24) mean that the graphs $S_{z}$ of $z(x)$ and $S_{w}$ of $w(p)$ are given by

$$
\begin{aligned}
& S_{z}=\partial\left(\bigcup_{p \in \bar{T}} K_{p, w(p)}\right), \\
& S_{w}=\partial\left(\bigcup_{x \in \bar{\Omega}} H_{x, z(x)}\right) .
\end{aligned}
$$

When the potential $V$ is concave we have similar characterizations of $S_{z}$ and $S_{w}$ with $\bigcup$ in (29), (30) replaced by $\bigcap$.

Remark 3.1. It follows from (23), (24) that when $V(x)$ is convex then for any $x \in \Omega$ the path taken by the light ray through the reflector system is the shortest among all possible paths (not necessarily satisfying the reflection law) that go from $(x, 0)$ to $(x, z(x))$, then to some $(p, w(p))$ and then to $(p, d)$. Of course, the shortest path satisfies the reflection law and $p=P(x)$. For concave $V$ the corresponding light path is the longest as it follows from (25), (26). Thus the characterizations (23), (24) and (25), (26) are variants of the Fermat principle.

\section{Weak solutions of Problem I}

We use the geometric characterizations of reflectors in section 3 to define weak solutions to Problem I. Let $\Omega$ and $T$ be two bounded domains on the 
hyperplane $\alpha$ and $\beta$ a fixed positive number.

Definition 4.1. A pair $(z, w) \in C(\bar{\Omega}) \times C(\bar{T})$ is called a two-reflector of type $A$ if

$$
\begin{aligned}
& z(x)=\sup _{p \in \bar{T}} k_{p, w(p)}(x), x \in \bar{\Omega}, \\
& w(p)=\inf _{x \in \bar{\Omega}} h_{x, z(x)}(p), p \in \bar{T},
\end{aligned}
$$

where $k_{p, w(p)}(x)$ and $h_{x, z(x)}(p)$ are defined by (27) and (28). Similarly, a pair $(z, w) \in C(\bar{\Omega}) \times C(\bar{T})$ is called a two-reflector of type $B$ if

$$
\begin{aligned}
& z(x)=\inf _{p \in \bar{T}} k_{p, w(p)}(x), x \in \bar{\Omega}, \\
& w(p)=\sup _{x \in \bar{\Omega}} h_{x, z(x)}(p), p \in \bar{T} .
\end{aligned}
$$

To avoid repetitions, we consider below only two-reflectors of type A. The changes that need to be made to deal with two-reflectors of type B are straight forward and are omitted.

It will be convenient to construct the following extensions $z^{*}$ of the function $z$ and $w^{*}$ of $w$ to the entire $\alpha$. For a pair $(z, w)$ as in definition 4.1 let

$$
V(x)=\frac{x^{2}}{2}+\beta z(x)-\frac{\beta^{2}}{2} \quad \text { and } \quad Q(x, p)=\langle x, p\rangle+\beta w(p)-\frac{p^{2}}{2} .
$$

It follows from (31) that

$$
V(x)=\sup _{p \in \bar{T}} Q(x, p), \quad x \in \bar{\Omega} .
$$

That is, $V$ is convex and continuous over $\bar{\Omega}$. Furthermore, since $\bar{T}$ is bounded, the graph $S_{V}$ has no vertical supporting hyperplanes. For any fixed $p \in \bar{T}$ define the half-space

$$
Q^{+}(p)=\left\{(x, Z) \in \alpha \times \mathbb{R}^{1} \mid Z \geq Q(x, p)\right\} .
$$

Then

$$
S_{V^{*}}=\partial\left(\bigcap_{p \in \bar{T}} Q^{+}(p)\right)
$$


is a graph of a convex function $V^{*}$ defined for all $x \in \alpha$. Note that $V^{*}(x)=$ $V(x)$ when $x \in \bar{\Omega}$. We now define an extension of $z$ by putting

$$
z^{*}(x)=\frac{1}{\beta}\left[-\frac{x^{2}}{2}+V^{*}(x)+\frac{\beta^{2}}{2}\right] .
$$

Similarly, for any fixed $x \in \bar{\Omega}$ we let

$$
R^{+}(x)=\left\{(p, Z) \in \alpha \times \mathbb{R}^{1} \mid Z \geq R(x, p)\right\}
$$

where

$$
R(x, p)=\langle x, p\rangle-\beta z(x)-\frac{x^{2}}{2}, \quad p \in \alpha
$$

Then the function

$$
U^{*}(p)=\sup _{x \in \bar{\Omega}} R(x, p), \quad p \in \alpha,
$$

is defined. It is also convex. It follows from (32) that for $p \in \bar{T}$

$$
U^{*}(p)=\frac{p^{2}}{2}-\beta w(p)-\frac{\beta^{2}}{2}(\equiv U(p)) .
$$

The corresponding extension of $w$ we define as

$$
w^{*}(p)=\frac{1}{\beta}\left[\frac{p^{2}}{2}-U^{*}(p)-\frac{\beta^{2}}{2}\right], \quad p \in \alpha .
$$

Lemma 4.2. The function $V^{*}$ is uniformly Lipschitz on $\alpha$ with Lipschitz constant $\max _{\bar{T}}|p|$. Also, $U^{*}(p), p \in \alpha$, is uniformly Lipschitz on $\alpha$ with Lipschitz constant $\max _{\bar{\Omega}}|x|$. In addition, $z \in \operatorname{Lip}(\bar{\Omega})$ and $w \in \operatorname{Lip}(\bar{T})$ with the Lipschitz constant $\leq \frac{\sup _{(x, p) \in \bar{\Omega} \times \bar{T}}|x-p|}{\beta}$.

Proof. By our convention the normal vector to a plane $Q(x, p)$ (when $p$ is fixed) is given by $(-p, 1)$. It follows from definition of $V^{*}$ that $S_{V^{*}}$ has no supporting hyperplanes with normal $(-p, 1)$ such that $p \notin \bar{T}$. Since $T$ is bounded, this implies the first statement of the lemma. The statements regarding $U^{*}$ are established by similar arguments. From these properties of $V^{*}$ it follows that the function $z^{*}$ is continuous on $\alpha$ and Lipschitz on any compact subset of $\alpha$. Similar properties hold also for $w^{*}$.

Now we estimate the Lipschitz constant for $z$ on $\bar{\Omega}$. Let $(z, w)$ be a tworeflector of type A. Let $x, x^{\prime} \in \bar{\Omega}$ and let $z\left(x^{\prime}\right) \geq z(x)$. (If the opposite 
inequality holds we relabel $x$ and $x^{\prime}$.) Fix some small $\varepsilon>0$. It follows from (31), (32) that there exists a $p^{\prime} \in \bar{T}$ such that $z\left(x^{\prime}\right) \leq k_{p^{\prime}, w\left(p^{\prime}\right)}\left(x^{\prime}\right)+\varepsilon$. Then

$$
\begin{aligned}
\left|z\left(x^{\prime}\right)-z(x)\right| & \leq k_{p^{\prime}, w\left(p^{\prime}\right)}\left(x^{\prime}\right)-z(x)+\varepsilon \leq k_{p^{\prime}, w\left(p^{\prime}\right)}\left(x^{\prime}\right)-k_{p^{\prime}, w\left(p^{\prime}\right)}(x)+\varepsilon \\
& \leq \sup _{x \in \bar{\Omega}}\left|\nabla k_{p^{\prime}, w\left(p^{\prime}\right)}(x)\right|\left|x^{\prime}-x\right|+\varepsilon=\frac{1}{\beta} \sup _{s \in \bar{\Omega}}\left|s-p^{\prime}\right|\left|x^{\prime}-x\right|+\varepsilon \\
& \leq \frac{1}{\beta} \sup _{s \in \bar{\Omega}, p \in \bar{T}}|s-p|\left|x^{\prime}-x\right|+\varepsilon .
\end{aligned}
$$

Letting $\varepsilon \longrightarrow 0$, we obtain the statement regarding Lipschitz constant for $z$. The same statement regarding $w$ and two-reflectors of type B are proved similarly.

Next, we define the analogue of the ray tracing map $P$ for a tworeflector. For that we need to recall the notion of the normal map [1], p. 114. Let $u: G \rightarrow \mathbb{R}^{1}$ be an arbitrary convex function defined on domain $G \subset \alpha$ and $S_{u}$ its graph. For $x_{0} \in G$ let

$$
Z-u\left(x_{0}\right)=\left\langle p, x-x_{0}\right\rangle
$$

be a hyperplane with normal $(-p, 1)$ supporting to $S_{u}$ at $\left(x_{0}, u\left(x_{0}\right)\right)$. The normal map $\nu_{u}: G \rightarrow \alpha$ at $x_{0}$ is defined as

$$
\nu_{u}\left(x_{0}\right)=\bigcup\{p\}
$$

where the union is taken over all hyperplanes supporting to $S_{u}$ at $\left(x_{0}, u\left(x_{0}\right)\right)$.

Definition 4.3. Let $(z, w)$ be a two reflector of type A. For $x \in \alpha$ we put

$$
\tilde{P}(x)=\nu_{V^{*}}(x) .
$$

For reflectors of type B the ray tracing map is defined similarly with the use of function $-U^{*}$. In general, $\tilde{P}$ may be multivalued.

Lemma 4.4. Let $(z, w)$ be a two-reflector of type $A$ and $z^{*}$ and $w^{*}$ their respective extensions as above. Then $\tilde{P}(x) \in \bar{T}$ for all $x \in \alpha$. In addition, for any $p \in \bar{T}$ the set $\{x \in \bar{\Omega} \mid \tilde{P}(x)=p\} \neq \emptyset$. Furthermore, for any $x \in \bar{\Omega}$

$$
\tilde{P}(x)=\left\{\text { all } p \in \bar{T} \mid w(p)=h_{x, z(x)}(p)\right\} .
$$


Proof. Let $x \in \alpha$ and $Q(x, p)$ a supporting hyperplane to $V^{*}$ at $\left(x, V^{*}(x)\right)$. Then the normal $p$ is in $\nu_{V^{*}}(x)$. On the other hand, by definition of $V^{*}, S_{V^{*}}$ has only supporting hyperplanes with normals in $\bar{T}$. Hence, $\tilde{P}(x) \subset \bar{T}$.

Let $p \in \bar{T}$ and $Q(x, p)$ a supporting hyperplane to $S_{V^{*}}$. We need to show that there is an $x \in \bar{\Omega}$ such that $p \in \tilde{P}(x)$. By (35) and (32) we have for any $x \in \bar{\Omega}$

$$
V(x)-Q(x, p)=\beta\left(h_{x, z(x)}(p)-w(p)\right) \geq 0 .
$$

By (32) there exists an $x \in \bar{\Omega}$ such that $V(x)-Q(x, p)=0$. This implies the remaining two statements of the lemma.

Remark 4.5. It follows from definition that $\tilde{P}$ is multivalued at points $x$ where $S_{V^{*}}$ has more than one supporting hyperplane. At such $x$ the function $z^{*}$ is not differentiable. Let $\left(x_{0}, z^{*}\left(x_{0}\right)\right)$ be one such point. Then

$$
\tilde{P}\left(x_{0}\right)=\left\{p \in \alpha \mid Q(x, p) \text { is supporting to } S_{V^{*}} \text { at }\left(x_{0}, V^{*}\left(x_{0}\right)\right)\right\} .
$$

In other words, a light ray labeled by $x_{0} \in \Omega$ that hits a point where the first reflector has a singular point will split into a cone of light rays. These rays will generate a subset on the paraboloid $h_{x_{0}, z\left(x_{0}\right)}(p)$ whose projection on $\alpha$ is $\tilde{P}\left(x_{0}\right)$. This is consistent with the physical interpretation of diffraction at singularities of this type [5].

Remark 4.6. Since $V^{*}$ is convex, by Rademacher's theorem, the Lebesgue measure of the set of singular points on $S_{V^{*}}$ is zero. Thus, $\tilde{P}(x)$ is singlevalued almost everywhere in $\alpha$. Furthermore, the functions $z\left(z^{*}\right)$ is a difference of two convex functions, and therefore, it is differentiable almost everywhere in $\Omega(\alpha)$. The same is true for $w$ and $w^{*}$. It follows then from the definitions of $\tilde{P}$ and $V$ that for almost all $x \in \Omega$

$$
\tilde{P}(x)=\nabla V=x+\beta \nabla z(x),
$$

A similar property holds also for the function $w$.

Lemma 4.7. If $(z, w)$ is a two-reflector of type $A$ then for all $x \in \bar{\Omega}, p \in \bar{T}$

$$
z(x)-w(p) \geq \frac{1}{2 \beta}\left(\beta^{2}-|x-p|^{2}\right) .
$$

In addition, for almost all $x \in \Omega$ there exists a unique $p \in \bar{T}$ such that $p=\tilde{P}(x)$ and (38) in this case is an equality. 
Proof. The lemma follows from (31), (32), Remark 4.6 and Lemma 4.4.

Define the inverse of $\tilde{P}$ for $p \in \bar{T}$ as

$$
\tilde{P}^{-1}(p)=\{x \in \alpha \mid p \in \tilde{P}(x)\} .
$$

Theorem 4.8. Let $\mathcal{B}$ be the $\sigma$-algebra of Borel subsets of $T$. Let $(z, w)$ be a two-refllector of type $A$. For any set $\tau \in \mathcal{B}$ the set $\tilde{P}^{-1}(\tau)$ is measurable relative to the standard Lebesgue measure on $\alpha$. In addition, for any nonnegative locally integrable function $I$ on $\alpha$ the function

$$
\mathcal{L}(\tau)=\int_{\tilde{P}^{-1}(\tau)} I(x) d x
$$

is a non-negative completely additive measure on $\mathcal{B}$.

Proof. The proof of this theorem is completely analogous to the proofs of Theorems 9 and 16 in [9].

Lemma 4.9. Let $\Omega$ and $T$ be two bounded domains on $\alpha$ and $I$ a nonnegative integrable function on $\Omega$ extended to entire $\alpha$ by setting $I(x) \equiv 0$ for $x \in \alpha \backslash \Omega$. Let $(z, w)$ be a two-reflector of type $A$ or $B$. Then for any continuous function $h$ on $\bar{T}$ we have the following change of variable formula

$$
\int_{T} h(p) \mathcal{L}(d p)=\int_{\Omega} h(\tilde{P}(x)) I(x) d x .
$$

Proof. In the integral on the right $h(\tilde{P}(x))$ is discontinuous only where $\tilde{P}$ is not single valued, that is, on the set of measure zero. Thus, the integral on the right is well defined.

We may assume that $\int_{\Omega} I(x) d x>0$; otherwise, the statement is trivial. Fix some small $\varepsilon>0$ and a positive integer N. Partition the interval $[\min h(p), \max h(p)]$ into sub-intervals $S_{1}, \ldots, S_{N}$ of length $<\varepsilon / \int_{\Omega} I(x) d x$ and let $h_{i} \in S_{i}$. Put $\tau_{i}=\left\{p \in T \mid h(p) \in S_{i}\right\}$. Then for sufficiently large $N$

$$
\left|\int_{T} h(p) \mathcal{L}(d p)-\sum h_{i} \mathcal{L}\left(\tau_{i}\right)\right|<\varepsilon
$$

For any $i, j=1, \ldots, N, i \neq j$, meas $\left(\tilde{P}^{-1}\left(\tau_{i}\right) \bigcap \tilde{P}^{-1}\left(\tau_{j}\right)\right)=0$ (see Remark 4.6). Hence,

$$
\left|\int_{\Omega} h(\tilde{P}(x)) I(x) d x-\sum h_{i} \int_{\tilde{P}^{-1}\left(\tau_{i}\right)} I(x) d x\right|<\varepsilon .
$$


This, together with the previous inequality, imply

$$
\left|\int_{\Omega} h(\tilde{P}(x)) I(x) d x-\sum h_{i} \mathcal{L}\left(\tau_{i}\right)\right|<2 \varepsilon .
$$

Definition 4.10. A two-reflector $(z, w)$ of type A ( B) is called a weak solution of type A (B) of the two-reflector problem I if the map $\tilde{P}: \bar{\Omega} \rightarrow \bar{T}$ is onto and for any Borel set $\tau \subseteq T$

$$
\mathcal{L}(\tau)=\int_{\tau} L(p) d p
$$

Using Lemma 4.9 and this definition we obtain

Lemma 4.11. Let $(z, w)$ be a weak solution of type $A(B)$ of the two-reflector problem I. Then for any continuous function $h$ on $\bar{T}$

$$
\int_{T} h(p) L(p) d p=\int_{\Omega} h(\tilde{P}(x)) I(x) d x .
$$

\section{A variational problem and weak solutions of the two-reflector problem}

As before, we consider here only the case of two-reflectors of type A. We comment on the case of two-reflectors of type B in section 7 . Let, as before, $l$ and $d$ be the given parameters of the system, and $\beta=l-d$. Let $\zeta \in C(\bar{\Omega})$ and $\omega \in C(\bar{T})$. With any such pair $(\zeta, \omega)$ we associate a "quasi-reflector" system in which the light path is defined as follows. Let $(x, p) \in \bar{\Omega} \times \bar{T}$ and let $(x, \zeta(x))$ be the point where the horizontal ray emanating from $(x, 0)$ hits the graph of $\zeta$. Let $P_{2}$ be the point where the horizontal ray begins in order to terminate in $(p, d)$. The "light" path is the polygon $\overline{(x, 0) P_{1} P_{2}(p, d)}$; see Fig. 2. The length of this path is

$$
l(\zeta, \omega, x, p)=\zeta(x)+\sqrt{(\zeta(x)-\omega(p))^{2}+|x-p|^{2}}+d-\omega(p) .
$$

The class of admissible pairs is defined as

$$
\operatorname{Adm}(\Omega, T)=\{(\zeta, \omega) \in C(\bar{\Omega}) \times C(\bar{T}) \mid l(\zeta, \omega, x, p) \geq l \forall(x, p) \in \bar{\Omega} \times \bar{T}\}
$$




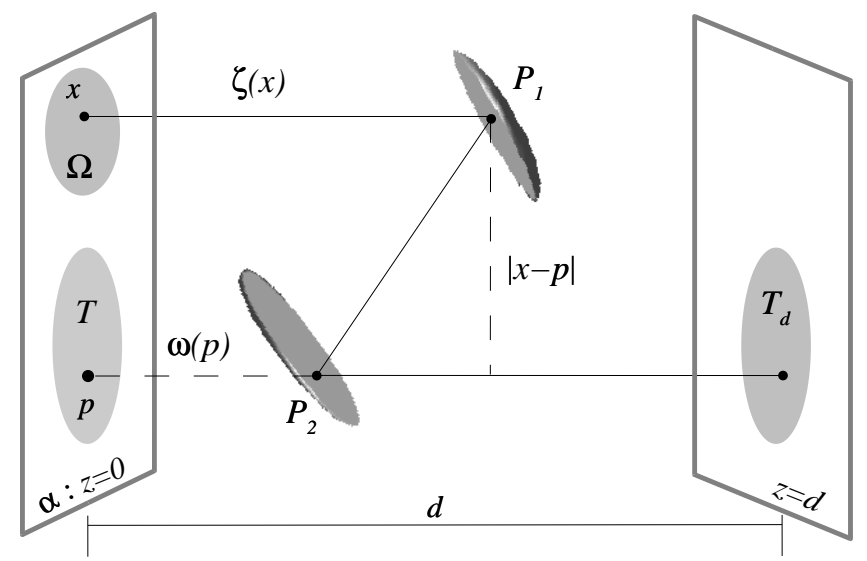

Figure 2: Definition of $l(\zeta, \omega, x, p)$.

By construction, a pair $(\zeta, \omega) \in C(\bar{\Omega}) \times C(\bar{T})$ lies in $A d m(\Omega, T)$ if and only if for all $x \in \bar{\Omega}, p \in \bar{T}$

$$
\zeta(x) \geq k_{p, \omega(p)}(x), \quad \omega(p) \leq h_{x, \zeta(x)}(p),
$$

or, equivalently, if and only if

$$
\zeta(x)-\omega(p) \geq \frac{1}{2 \beta}\left(\beta^{2}-|x-p|^{2}\right)
$$

for all $x \in \bar{\Omega}, p \in \bar{T}$. It follows from (31), (32) that a two-reflector of type A is an admissible pair.

The following functional is central to our investigation.

Let $I$ and $L$ be two nonnegative integrable functions on $\Omega$ and $T$, respectively, satisfying the energy conservation law (8). For $(\zeta, \omega) \in \operatorname{Adm}(\Omega, T)$ put

$$
\mathcal{F}(\zeta, \omega)=\int_{\Omega} \zeta(x) I d x-\int_{T} \omega(p) L d p
$$

Clearly, $\mathcal{F}$ is linear and bounded on $C(\bar{\Omega}) \times C(\bar{T})$ with respect to the norm $\max \left\{\|\zeta\|_{\infty},\|\omega\|_{\infty}\right\}$. Geometrically, $\mathcal{F}(\zeta, \omega)$ is proportional to the mean horizontal distance between the points of the two graphs, the average being weighted by the intensities.

We consider now the following 
Problem II. Minimize $\mathcal{F}$ on $\operatorname{Adm}(\Omega, T)$.

Proposition 5.1. [2, 3, 4] There exist $(z, w) \in \operatorname{Adm}(\Omega, T)$ such that

$$
\mathcal{F}(z, w)=\inf _{(\zeta, \omega) \in \operatorname{Adm}(\Omega, T)} \mathcal{F}(\zeta, \omega) .
$$

We may further assume that the pair (z,w) satisfies the conditions (31), (32) for a type A reflector system.

Proof. It follows from (42) that one can restrict the search for minimizers to two-reflectors $(\zeta, \omega)$ of type A. Also, because of $(8 \mathcal{F}(\zeta, \omega)$ is invariant under translations $\zeta \mapsto \zeta+\rho, \omega \mapsto \omega+\rho$ for a constant $\rho \in \mathbb{R}$. Hence, it is sufficient to consider only two-reflectors $(\zeta, \omega)$ for which $\zeta\left(x_{0}\right)=0$ for some $x_{0} \in \bar{\Omega}$.

By Lemma 4.2, $\zeta$ and $\omega$ are uniformly Lipschitz with the Lipschitz constant $K=\sup _{x \in \Omega, p \in T}|x-p| / \beta$. It follows that for all $x \in \Omega$

$$
|\zeta(x)|=\left|\zeta(x)-\zeta\left(x_{0}\right)\right| \leq K \operatorname{diam} \Omega .
$$

For all $p \in \bar{T}$ we have

$$
\omega(p) \leq h_{x_{0}, \zeta\left(x_{0}\right)}(p)=\frac{1}{2 \beta}\left(\left|x_{0}-p\right|^{2}-\beta^{2}\right) \leq \max _{q \in \bar{T}} \frac{1}{2 \beta}\left(\left|x_{0}-q\right|^{2}-\beta^{2}\right) .
$$

Finally, since $h_{x, \zeta(x)}(p)=\left(|x-p|^{2}-\beta^{2}\right) / 2 \beta+\zeta(x) \geq-\beta / 2-K \operatorname{diam} \Omega$ for all $x \in \bar{\Omega}$, we also get the lower bound

$$
\omega(p)=\inf _{x \in \Omega} h_{x, \zeta(x)}(p) \geq-\frac{\beta}{2}-K \operatorname{diam} \Omega .
$$

Therefore, the maps $\zeta$ and $\omega$ are also uniformly bounded. By the ArzelàAscoli theorem, and because $\mathcal{F}$ is continuous, the infimum of $\mathcal{F}$ is achieved at some two-reflector pair $(z, w)$.

We now show that weak solutions of Problem I and solutions of the Problem II are the same. This establishes existence of weak solutions to Problem I.

Theorem 5.2. Let $z \in C(\bar{\Omega}), w \in C(\bar{T})$ be a two-reflector of type A. Then the following statements are equivalent.

(i) $(z, w)$ minimizes $\mathcal{F}$ in $\operatorname{Adm}(\Omega, T)$.

(ii) $(z, w)$ is a weak solution of type A of the two-reflector Problem I. 
Proof. (ii) $\Rightarrow(\mathrm{i})$. Let $(\zeta, \omega) \in \operatorname{Adm}(\Omega, T)$. Then by (43) and Lemma 4.7 for almost all $x \in \Omega$

$$
\zeta(x)-\omega(\tilde{P}(x)) \geq \frac{1}{2 \beta}\left(\beta^{2}-|x-\tilde{P}(x)|^{2}\right)=z(x)-w(\tilde{P}(x)) .
$$

Integrating this inequality we get

$$
\int_{\Omega} \zeta(x) I d x-\int_{\Omega} \omega(\tilde{P}(x)) I d x \geq \int_{\Omega} z(x) I d x-\int_{\Omega} w(\tilde{P}(x)) I d x .
$$

Using Lemma 4.11, we get

$\mathcal{F}(\zeta, \omega)=\int_{\Omega} \zeta(x) I d x-\int_{T} \omega(p) L d p \geq \int_{\Omega} z(x) I d x-\int_{T} w(p) L d x=\mathcal{F}(z, w)$.

Since $(\zeta, \omega)$ was arbitrary, we are done.

(i) $\Rightarrow$ (ii). Let $\tilde{P}$ denote the ray tracing map for the pair $(z, w)$. It must be shown that $\int_{\tilde{P}^{-1}(\tau)} I(x) d x=\int_{\tau} L(p) d p$ for all Borel sets $\tau \subseteq T$. We will prove that this is the Euler-Lagrange equation for the functional $\mathcal{F}$.

It is sufficient to establish this for the case when $\tau$ is an open ball with center $p_{0} \in T$ and radius $r>0$ contained in $T$. For $i=1,2, \cdots$ define for $p \in \alpha$

$$
\chi_{i}(p)= \begin{cases}1, & \text { if }\left|p-p_{0}\right|<r-\frac{1}{i} \\ i\left(r-\left|p-p_{0}\right|\right), & \text { if } r-\frac{1}{i} \leq\left|p-p_{0}\right|<r \\ 0, & \text { if }\left|p-p_{0}\right| \geq r .\end{cases}
$$

Then $\chi_{i}$ is continuous on $\alpha, 0 \leq \chi_{i} \leq 1$, and the sequence $\left\{\chi_{i}(p): p \in \alpha\right\}_{i=1}^{\infty}$ converges on $\alpha$ pointwise to the characteristic function of $\tau \chi_{\tau}(p)$.

Fix some $i$ and for $\varepsilon \in(-1,1)$ put

$$
\begin{aligned}
& w_{\varepsilon}(p)=w(p)+\varepsilon \cdot \chi_{i}(p) \\
& z_{\varepsilon}(x)=\sup _{p \in \bar{T}} k_{p, w_{\varepsilon}(p)}(x)=\sup _{p \in \bar{T}}\left\{\frac{1}{2 \beta}\left(\beta_{0}^{2}-|x-p|^{2}\right)+w_{\varepsilon}(p)\right\} .
\end{aligned}
$$

By construction, the pair $\left(z_{\varepsilon}, w_{\varepsilon}\right)$ satisfies the condition (43), with $\zeta$ replaced by $z_{\varepsilon}$ and $\omega$ repaced by $w_{\varepsilon}$. We show now that $z_{\varepsilon}$ belongs to $\operatorname{Lip}(\bar{\Omega})$. Let 
$x, x^{\prime} \in \bar{\Omega}$ and let $z\left(x^{\prime}\right) \geq z(x)$. (If the opposite inequality holds we relabel $x$ and $x^{\prime}$.) Let $p^{\prime} \in \bar{T}$ be such that $z_{\varepsilon}\left(x^{\prime}\right)=k_{p^{\prime}, w_{\varepsilon}\left(p^{\prime}\right)}\left(x^{\prime}\right)$. Then

$$
\begin{aligned}
\left|z_{\varepsilon}\left(x^{\prime}\right)-z_{\varepsilon}(x)\right| & =k_{p^{\prime}, w_{\varepsilon}\left(p^{\prime}\right)}\left(x^{\prime}\right)-z_{\varepsilon}(x) \\
& \leq k_{p^{\prime}, w_{\varepsilon}\left(p^{\prime}\right)}\left(x^{\prime}\right)-k_{p^{\prime}, w_{\varepsilon}\left(p^{\prime}\right)}(x) \\
& \leq \sup _{s \in \bar{\Omega}}\left|\nabla k_{p^{\prime}, w_{\varepsilon}\left(p^{\prime}\right)}(s)\right|\left|x^{\prime}-x\right|=\frac{1}{\beta} \sup _{s \in \bar{\Omega}}\left|s-p^{\prime}\right|\left|x^{\prime}-x\right| \\
& \leq \frac{1}{\beta} \sup _{s \in \bar{\Omega}, p \in \bar{T}}|s-p|\left|x^{\prime}-x\right| .
\end{aligned}
$$

Hence, $z_{\varepsilon}$ is continuous and $\left(z_{\varepsilon}, w_{\varepsilon}\right) \in \operatorname{Adm}(\Omega, T)$.

Now let $x \in \bar{\Omega}$. For each $\varepsilon$ let $p_{\varepsilon}$ be a point in $\bar{T}$ such that $z_{\varepsilon}(x)=$ $k_{p_{\varepsilon}, w_{\varepsilon}\left(p_{\varepsilon}\right)}(x)$. (This choice, of course, may not be unique.) Then

$$
\begin{aligned}
z_{\varepsilon}(x)-z(x) & =k_{p_{\varepsilon}, w_{\varepsilon}\left(p_{\varepsilon}\right)}(x)-z(x) \leq k_{p_{\varepsilon}, w_{\varepsilon}\left(p_{\varepsilon}\right)}(x)-k_{p_{\varepsilon}, w\left(p_{\varepsilon}\right)}(x) \\
& =w_{\varepsilon}\left(p_{\varepsilon}\right)-w\left(p_{\varepsilon}\right)=\varepsilon \chi_{i}\left(p_{\varepsilon}\right) .
\end{aligned}
$$

Similarly, if $p \in \tilde{P}(x)$, then

$$
\begin{aligned}
z_{\varepsilon}(x)-z(x) & =z_{\varepsilon}(x)-k_{p, w(p)}(x) \geq k_{p, w_{\varepsilon}(p)}(x)-k_{p, w(p)}(x) \\
& =\varepsilon \chi_{i}(p) .
\end{aligned}
$$

Therefore,

$$
-|\varepsilon| \leq \varepsilon \chi_{i}(p) \leq z_{\varepsilon}(x)-z(x) \leq \varepsilon \chi_{i}\left(p_{\varepsilon}\right) \leq|\varepsilon|
$$

for all $x \in \bar{\Omega}$. In particular, $z_{\varepsilon}$ converges uniformly to $z$ on $\bar{\Omega}$ as $\varepsilon \rightarrow 0$.

Now consider those $x \in \Omega$ for which the ray tracing map $\tilde{P}$ is singlevalued. This is the case for almost all $x \in \Omega$. We claim that $p_{\varepsilon}=\tilde{P}_{\varepsilon}$, where $\tilde{P}_{\varepsilon}$ denotes the ray tracing map for $\left(z_{\varepsilon}, w_{\varepsilon}\right)$, converge to $p$ as $\varepsilon \rightarrow 0$.

Suppose this is not true. Then there is a sequence $\left\{p_{\varepsilon_{j}}\right\}, j=1,2, \ldots$ with $\varepsilon_{j} \rightarrow 0$ as $j \rightarrow \infty$ and a constant $\eta>0$ such that

$$
\left|p-p_{\varepsilon_{j}}\right|>\eta \text { for all } j \text {. }
$$

Let $z^{\prime}(x)=\max _{p^{\prime} \in \bar{T},\left|p^{\prime}-p\right| \geq \eta} k_{p^{\prime}, w\left(p^{\prime}\right)}(x)$. Note that the maximum in the definition of $z^{\prime}$ is attained. Since $p$ is the unique point in $\bar{T}$ such that $z(x)=k_{p, w(p)}(x)$, it follows that $z^{\prime}<z(x)$. Therefore, for all $j$

$$
\begin{aligned}
z(x)-z_{\varepsilon_{j}}(x) & =z(x)-k_{p_{\varepsilon_{j}}, w_{\varepsilon_{j}}\left(p_{\varepsilon_{j}}\right)}(x)=z(x)-k_{p_{\varepsilon_{j}}, w\left(p_{\varepsilon_{j}}\right)}(x)+w\left(p_{\varepsilon_{j}}\right)-w_{\varepsilon_{j}}\left(p_{\varepsilon_{j}}\right) \\
& =z(x)-k_{p_{\varepsilon_{j}}, w\left(p_{\varepsilon_{j}}\right)}(x)-\varepsilon_{j} \chi_{i}\left(p_{\varepsilon_{j}}\right) \\
& \geq z(x)-z^{\prime}-\left|\varepsilon_{j}\right| .
\end{aligned}
$$


This contradicts to the fact that $z_{\varepsilon_{j}}(x)$ converges to $z(x)$ as $j \rightarrow \infty$. Hence, $p_{\varepsilon} \rightarrow p$ if $\varepsilon \rightarrow 0$.

It follows from (44) that

$$
\left|\frac{z_{\varepsilon}(x)-z(x)}{\varepsilon}-\chi_{i}(p)\right| \leq\left|\chi_{i}\left(p_{\varepsilon}\right)-\chi_{i}(p)\right| .
$$

Letting $\varepsilon \rightarrow 0$ and using the continuity of $\chi_{i}$, we conclude that for almost all $x \in \Omega$

Then

$$
\left.\frac{d}{d \varepsilon}\right|_{\varepsilon=0} z_{\varepsilon}(x)=\chi_{i}(p)=\chi_{i}(\tilde{P}(x)) .
$$

$$
\left.\frac{d}{d \varepsilon}\right|_{\varepsilon=0} \int_{\Omega} z_{\varepsilon}(x) I(x) d x=\int_{\Omega} \chi_{i}(\tilde{P}(x)) I(x) d x .
$$

Since $\mathcal{F}$ has a minimum at $(z, w)$, we obtain

$$
0=\left.\frac{d}{d \varepsilon}\right|_{\varepsilon=0} \mathcal{F}\left(z_{\varepsilon}, w_{\varepsilon}\right)=\int_{\Omega} \chi_{i}(\tilde{P}(x)) I(x) d x-\int_{T} \chi_{i}(p) L(p) d p .
$$

Now let $i \rightarrow \infty$ in this equality. This is possible as $\chi_{i}(p) \rightarrow \chi_{\tau}(p)$ pointwise on $\alpha$ and therefore $\chi_{i}(\tilde{P}(x)) \rightarrow \chi_{\tau}(\tilde{P}(x))$ pointwise almost everywhere on $\Omega$. Then, noting that for almost all $x \in \Omega \quad \chi_{\tau}(\tilde{P}(x))=\chi_{\tilde{P}^{-1}(\tau)}(x)$, we obtain

$$
\begin{aligned}
\int_{\tau} L(p) d p & =\int_{T} \chi_{\tau}(p) L(p) d p=\int_{\Omega} \chi_{\tau}(\tilde{P}(x)) I(x) d x \\
& =\int_{\Omega} \chi_{\tilde{P}^{-1}(\tau)}(x) I(x) d x=\int_{\tilde{P}^{-1}(\tau)} I(x) d x .
\end{aligned}
$$

This completes the proof of the theorem.

\section{Connection between the two-reflector and Monge-Kantorovich problems}

In our notation, the Monge-Kantorovich mass transfer problem [2] can be formulated as follows. Consider the class of maps $P: \Omega \rightarrow T$ which are measure-preserving, that is, they satisfy the substitution rule

$$
\int_{\Omega} h(P(x)) I d x=\int_{T} h(p) L d p
$$

for all continuous functions $h$ on $\bar{T}$. Each such map is called a plan. 
Problem III. Minimize the quadratic transportation cost

$$
P \mapsto \frac{1}{2} \int_{\Omega}|x-P(x)|^{2} I d x .
$$

among all plans $P$.

Note that for weak solutions to the two-reflector Problem I the ray tracing map $\tilde{P}$ is a plan by Lemma 4.11. In fact, we have the following

Theorem 6.1. Let $(z, w)$ be a weak solution of type $A$ of the two-reflector Problem I. Let $\tilde{P}$ be the corresponding ray tracing map. Then $\tilde{P}$ minimizes the quadratic transportation cost (45) among all plans $P: \Omega \rightarrow T$, and any other minimizer is equal to $\tilde{P}$ almost everywhere on $\operatorname{supp}(I) \backslash\{x \in \bar{\Omega}: I(x)=$ $0\}$.

Proof. Let $P: \Omega \rightarrow T$ be any plan. Then by (38),

$$
z(x)-w(P(x)) \geq \frac{1}{2 \beta}\left(\beta^{2}-|x-P(x)|^{2}\right),
$$

for almost all $x \in \Omega$ and equality holds if and only if $P(x)=\tilde{P}(x)$. Integrating against $I d x$ and applying Lemma 4.11, we get

$$
\begin{aligned}
\frac{1}{2 \beta} \int_{\Omega}\left[\beta^{2}-\mid\right. & \left.x-\left.P(x)\right|^{2}\right] I d x \leq \int_{\Omega}[z(x)-w(P(x))] I d x \\
= & \int_{\Omega} z(x) I d x-\int_{T} w(p) L d p=\int_{\Omega}[z(x)-w(\tilde{P}(x))] I d x \\
= & \frac{1}{2 \beta} \int_{\Omega}\left[\beta^{2}-|x-\tilde{P}(x)|^{2}\right] I d x .
\end{aligned}
$$

This shows that $\tilde{P}$ is a minimizer of the transportation cost.

To show uniqueness, note that if equality holds in the integral inequality, then equality must hold in (46) for almost all $x \in \operatorname{supp}(I) \backslash\{I=0\}$. Therefore, $P \equiv \tilde{P}$ a.e. on $\operatorname{supp}(I) \backslash\{I=0\}$.

Remark 6.2. The functional $\mathcal{F}$ is the dual of the quadratic cost functional (45) $[2]$. 


\section{Existence and uniqueness of weak solutions to the two-reflector problem}

Theorem 7.1. There exist weak solutions of type $A$ and of type $B$ to the two-reflector Problem I. Furthermore, if $(z, w)$ and $\left(z^{\prime}, w^{\prime}\right)$ are two solutions of the same type with ray tracing maps $\tilde{P}$ and $\tilde{P}^{\prime}$, respectively, then

$$
\tilde{P}(x) \equiv \tilde{P}^{\prime}(x)
$$

for almost all $x \in \operatorname{supp}(I) \backslash\{x \in \Omega: I(x)=0\}$.

Proof. By Proposition 5.1 and Theorem 5.2 we know that Problem I has a solution. The only property that remains to be checked is that for that solution, $\tilde{P}: \bar{\Omega} \rightarrow \bar{T}$ is onto. But this follows from Lemma 4.4.

In order to prove uniqueness, let $(z, w)$ and $\left(z^{\prime}, w^{\prime}\right)$ be two solutions of type A, with ray tracing maps $\tilde{P}$ and $\tilde{P}^{\prime}$, respectively. Then by Proposition 6.1, both are minimizers of the quadratic Monge-Kantorovich cost functional, so that $\tilde{P}=\tilde{P}^{\prime}$ a.e. on $\operatorname{supp}(I) \backslash\{I=0\}$.

So far, existence and uniqueness of weak solutions to the two-reflector Problem I has been shown for weak solutions of type A. However, it is clear that a similar result holds for weak solutions of type B as well. Namely, we can define $\operatorname{Adm}_{+}(\Omega, T)$ as the space of all pairs of reflectors such that $l(\xi, \omega, x, p)$ is less than $l$, and then that $\mathcal{F}$ admits a maximizing pair on $\operatorname{Adm}_{+}(\Omega, T)$, which is a weak solution of the two-reflector problem I. This shows, in particular, that for such a solution the ray tracing map maximizes the quadratic transportation cost among all plans.

Corollary 7.2. Suppose that in addition to the assumptions in Theorem 7.1 the function $I>0$ in $\Omega$. Then there is a constant $\rho \in \mathbb{R}$ such that

$$
\begin{aligned}
& z^{\prime}(x)=z(x)+\rho \\
& w^{\prime}(p)=w(p)+\rho
\end{aligned}
$$

for all $x \in \bar{\Omega}$ and all $p \in \bar{T}$. In other words, weak solutions of type $A$ are unique on $\bar{\Omega}$ and $\bar{T}$ up to a translation of the reflector system, and the same result holds for type $B$ solutions.

Proof. We show this for weak solutions of type A. By the theorem, $\tilde{P}(x) \equiv$ $\tilde{P}^{\prime}(x)$ for all $x \in \Omega$. Note that by $(37), \nabla z(x)=\nabla z^{\prime}(x)$ for almost all $x \in \Omega$. 
It follows that there is a constant $\rho$ such that $z^{\prime}(x)=z(x)+\rho$ for all $x \in \bar{\Omega}$. Now, by definition of $w^{\prime}(p)$,

$$
w^{\prime}(p)=\inf _{x \in \bar{\Omega}} h_{x, z^{\prime}(x)}(p)=\inf _{x \in \bar{\Omega}} h_{x, z(x)}(p)+\rho=w(p)+\rho
$$

for all $p \in \bar{T}$.

\section{References}

[1] I. J. Bakelman. Convex Analysis and Nonlinear Geometric Elliptic Equations. Springer-Verlag, Berlin, 1994.

[2] Y. Brenier. Polar factorization and monotone rearrangement of vectorvalued functions. Comm. in Pure and Applied Math., 44:375-417, 1991.

[3] L.A. Caffarelli. Allocation maps with general cost functions. Partial Differential Equations and Applications, 177:29-35, 1996.

[4] W. Gangbo and R. J. McCann. Optimal maps in Monge's mass transport problem. C. R. Acad. Sci. Paris Sér. I Math., 321(12):1653-1658, 1995.

[5] J. B. Keller and R. M. Lewis. Asymptotic methods for partial differential equations: the reduced wave equation and maxwell's equations. Surveys in Applied Mathematics, ed. by J.B. Keller, D.M. McLaughlin, and G.C. Papanicolau, 1:1-82, 1995.

[6] P. H. Malyak. Two-mirror unobscured optical system for reshaping irradiance distribution of a laser beam. Applied Optics, 31(22):4377-4383, August 1992.

[7] V. Oliker and L.D. Prussner. A new technique for synthesis of offset dual reflector systems. In 10-th Annual Review of Progress in Applied Computational Electromagnetics, pages 45-52, 1994.

[8] V.I. Oliker. Mathematical aspects of design of beam shaping surfaces in geometrical optics. In Trends in Nonlinear Analysis, ed. by M. Kirkilionis, S. Krmker, R. Rannacher, F. Tomi, pages 191-222. SpringerVerlag, 2002. 
[9] V.I. Oliker. On the geometry of convex reflectors. PDE's, Submanifolds and Affine Differential Geometry, Banach Center Publications, 57:155$169,2002$.

[10] R. Schneider. Convex Bodies. The Brunn-Minkowski Theory. Cambridge Univ. Press, Cambridge, 1993. 